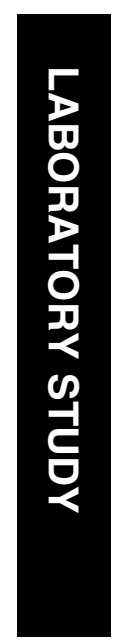

\section{Intraocular use of rituximab}

'Department of Ophthalmology, Mayo

Clinic, Rochester, MN, USA

${ }^{2}$ Dakota Eye Institute, Bismarck, ND, USA

${ }^{3}$ Department of Molecular Pharmacology and Experimental Therapeutics, Mayo Clinic, Scottsdale, AZ, USA

${ }^{4}$ Department of Neurology, Mayo Clinic, Rochester, MN, USA

${ }^{5}$ Department of Internal Medicine, Hematology Division, Mayo Clinic, Rochester, MN, USA

${ }^{6}$ Department of Pathology, Mayo Clinic, Rochester, MN, USA

Correspondence: JS Pulido, Department of

Ophthalmology,

Mayo Clinic,

200 First Street, SW,

Rochester, MN 55905, USA

Tel: + 15072843721 ;

Fax: + 15072844612

E-mail: pulido.jose@

mayo.edu

Received: 23 December 2006

Accepted in revised form:

1 March 2007

Published online: 20 April 2007

\begin{abstract}
Purpose To evaluate the toxicity of $\mathbf{1} \mathbf{m g}$ of intraocular rituximab and to present a small case-series of patients treated with intravitreal rituximab.

Methods Rituximab $(1 \mathrm{mg} / 0.1 \mathrm{ml})$ was injected in the vitreous of one eye of three Dutch-belted rabbits. Two animals were injected with balanced salt solution as controls. At 1 month the rabbits were killed and the eyes examined by light microscopy. Three patients (five eyes) with intraocular lymphoma were also treated with a $1 \mathrm{mg}$ injection of rituximab.

Results The treated rabbit eyes and the control eyes showed no light microscopic evidence of ocular toxicity at 1 month following injection. The five human eyes of three patients have shown no evidence of intraocular toxicity with a median follow-up time of 3.6 months (range 2.0-6.4 months). One patient received a total of four injections in the right eye and three injections in the left eye. Conclusion Intravitreal rituximab at a dose of $\mathbf{1} \mathrm{mg}$ does not appear to cause toxicity in rabbit eyes and in the five eyes of three patients. Eye (2007) 21, 1524-1527; doi:10.1038/sj.eye.6702804; published online 20 April 2007
\end{abstract}

Keywords: intraocular lymphoma; intraocular toxicity; primary central nervous system lymphoma; rituximab

\section{Introduction}

Rituximab is a humanized monoclonal mouse antibody that targets CD20-positive B cells and has been approved for systemic use in patients with B cell lymphoma. It has also been administered systemically as salvage therapy, by itself or in combination with temzolamide, for primary central nervous system (CNS) lymphoma. ${ }^{1,2}$
AS Kitzmann', JS Pulido', BG Mohney ${ }^{1}$, KH Baratz', T Grube², RJ Marler', MJ Donaldson', BP O'Neill4, PB Johnston ${ }^{5}$, KM Johnson', LE Dixon ${ }^{3}$, DR Salomao ${ }^{6}$ and JD Cameron

Primary CNS lymphoma is characterized by malignant lymphocytes that express certain B cell markers including CD20. Therefore, rituximab may be an ideal candidate for treating CNS lymphomas, but it may not penetrate the blood-brain or blood-retinal barriers. The currently available intravitreal chemotherapy for intraocular lymphoma is intraocular methotrexate. ${ }^{3}$ A recent study by Kim et al ${ }^{4}$ showed that intraocular injections of $1 \mathrm{mg}$ of rituximab had a half-life of 4.7 days in rabbits and did not appear to cause toxicity. Herein we confirm the lack of histologic toxicity of intraocular rituximab in animals and describe its use in three patients (five eyes).

\section{Methods}

Approval was obtained from both Institutional Animal Care and Use Committee and from Mayo Clinic Institutional Review Board. We certify that all applicable institutional and governmental regulations concerning the ethical use of human patients and animals were followed during this research.

Five Dutch-belted male rabbits (Harlan Laboratories, Indianapolis, IN, USA) were anaesthetized with $35 \mathrm{mg} / \mathrm{kg}$ of intramuscular ketamine hydrochloride (Fort Dodge Inc., Fort Dodge, IN, USA), $5 \mathrm{mg} / \mathrm{kg}$ of intramuscular xylazine hydrochloride (Phoenix Scientific Inc., St Joseph, MO, USA), and 1\% proparacaine hydrochloride ophthalmic drops (Allergan America, Hormigueros, Puerto Rico). Using a 30-gauge needle, three rabbits had one eye injected with intravitreal rituximab $1 \mathrm{mg} / 0.1 \mathrm{ml}$. For a control group, two rabbits were injected with balanced salt solution in the right eye. The noninjected left eyes also served as a control group. The animals were killed at 1 month with intravenous pentobarbital (Beuthanasia-D Special; Schering-Plough, Kenilworth, NJ, USA). Both eyes were enucleated, fixed with $2 \%$ paraformaldehyde, paraffin-embedded, sectioned, and stained with haematoxylin and 
eosin. The sections were evaluated by light microscopy by two masked pathologists.

\section{Results}

The histologic appearances of the three treated right eyes were no different from the untreated left eyes nor were there any differences from the balanced salt solution-injected right eyes (Figure 1). The retinas showed only mild vacuolization in the ganglion cell layer in both the treated and control eyes secondary to fixation artifact. There was no histologic evidence of toxicity to the retina, the optic nerve, or any other intraocular tissues in any of the sections examined.

\section{Case series}

\section{Case 1}

A 68-year-old woman presented with a complaint of decreased vision and floaters in the left eye for 2 months. She had a history of biopsy-proven primary CNS lymphoma that had been treated with whole-brain radiotherapy and CHOP (cyclophosphamide, doxorubicin, vincristine, prednisone) chemotherapy at an outside institution 3 years before her recent ocular symptoms.

On presentation, her corrected visual acuity was 20/30 in the right eye and 20/50 in the left. There were a few cells in the vitreous of the right eye and $1+$ cell in the vitreous of the left eye. The retina of the right eye showed a few flame-shaped intraretinal haemorrhages consistent with radiation retinopathy, and the fundus of the left eye showed two areas of subretinal white infiltrates

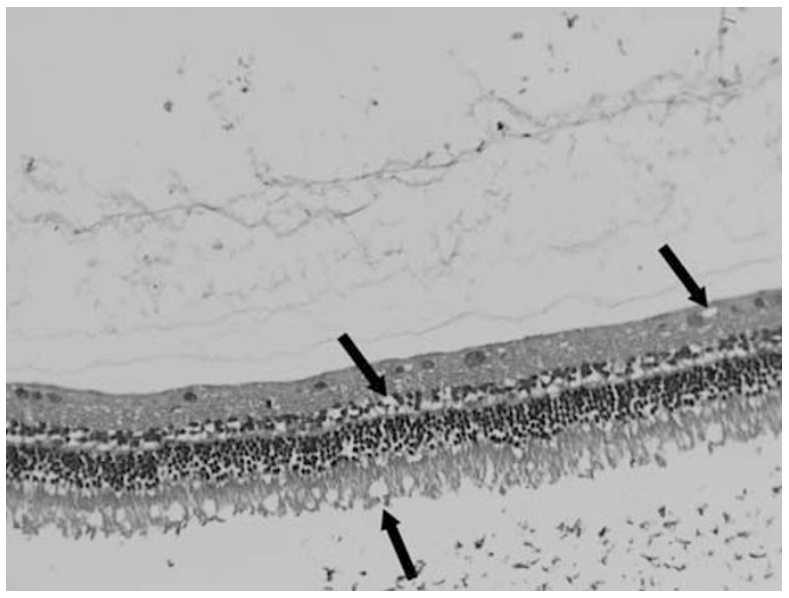

Figure 1 Photomicrograph of the retina of a representative rabbit eye following the injection of $1 \mathrm{mg}$ of rituximab 1 month earlier. There is mild fixation vacuolization (arrows). All the layers appear intact without evidence of retinal toxicity. (Haematoxylineosin stain, original magnification $\times 200$ )
(Figure 2). An MRI of the head showed no evidence of residual or recurrent primary CNS lymphoma, but did demonstrate diffuse cortical atrophy and white matter signal changes consistent with treatment-related neurotoxicity. There were signs of radiation cerebritis, but PET/CT also showed no evidence of cerebral disease. A 25-gauge vitrectomy and subretinal aspiration of the plaque of cells from one of the subretinal infiltrates in the left eye were performed, followed by intraocular endolaser around the subretinal lesion. Because of her pre-existing neurotoxicity and overall health, the oncologists and the family did not wish to use additional systemic chemotherapy. Instead, they wished to try a therapy that may be given less often than intraocular methotrexate. Methotrexate $200 \mu \mathrm{g} / 0.05 \mathrm{ml}$ (half the standard dose) and rituximab $1 \mathrm{mg} / 0.1 \mathrm{ml}$ were then given intravitreally. Histopathological examination of the vitrectomy specimen showed the presence of large, atypical lymphocytes, positive for CD20 consistent with large B-cell lymphoma.

The patient subsequently received intravitreal injections of rituximab in the right eye and repeated intravitreal injections of rituximab in the left eye, for a total of four injections in the right eye and three injections in the left eye. Approximately 3 months after the first intravitreal rituximab injection in the left eye, the patient underwent cataract surgery on the left eye. On last examination, 6.4 months after the initial injection in the left eye, the patient's visual acuity with correction was 20/50 and 20/30 in the right and left eyes, respectively. There were no vitreous cells in the right eye. In the left eye, there were only trace vitreous cells and there was scarring in the area of the subretinal infiltrate and biopsy (Figure 3). The patient has continued to show no clinical evidence of toxicity.

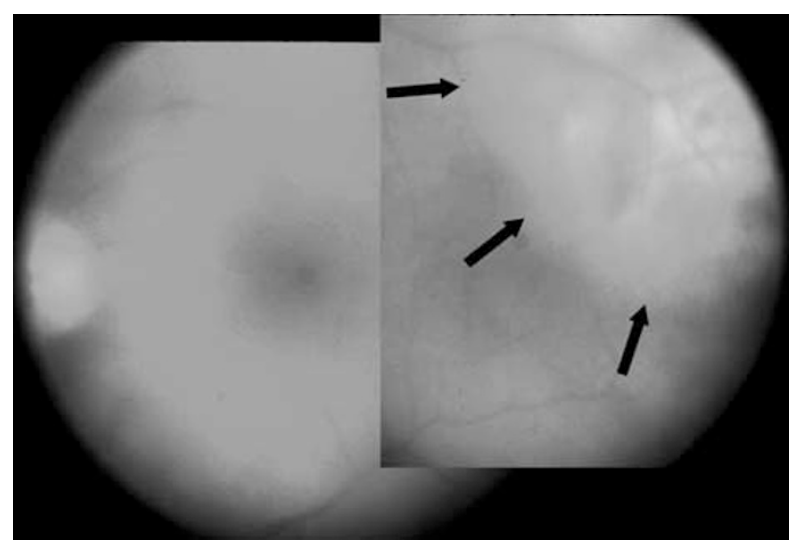

Figure 2 Fundus photograph of the patient in Case 1 before the intraocular injection of $1 \mathrm{mg}$ rituximab and $200 \mu \mathrm{g}$ of methotrexate. Note the retinal and subretinal involvement superotemporal to the fovea (arrows). The view is hazy due to vitreous cells. 


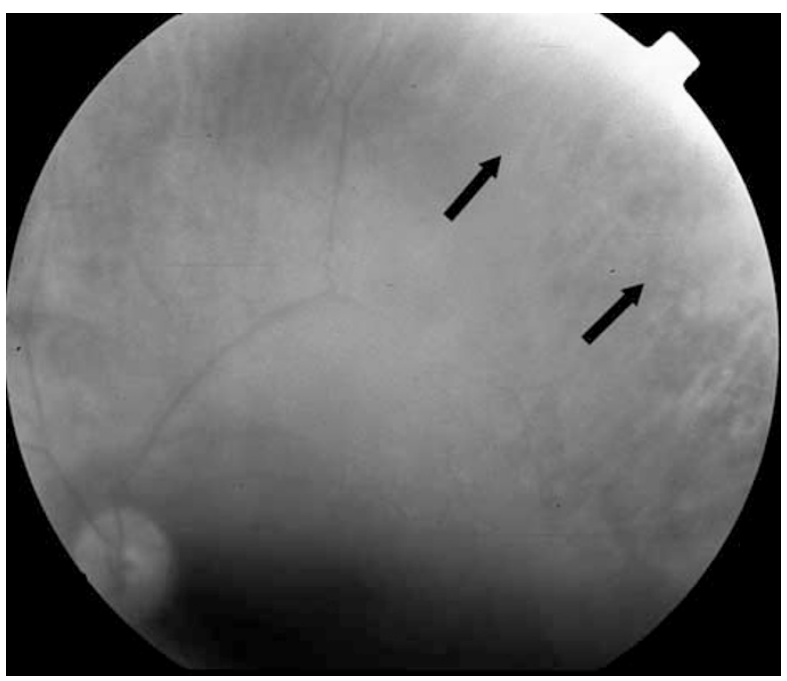

Figure 3 Fundus photograph of the patient in case 1, 6.4 months following the initial injection of methotrexate and rituximab. Note the area of scarring superotemporal to the fovea where the subretinal biopsy was performed (arrows). The view is clearer because there are very few media opacities.

\section{Case 2}

A 70-year-old man with a recent diagnosis of primary CNS lymphoma, based on positive brain biopsy and cerebral spinal fluid analysis, was referred to the Ophthalmology Department for blurry vision in the right eye. On initial examination, the patient's visual acuity with correction was 20/25 and 20/400 in the right and left eyes, respectively. The patient had a history of amblyopia in the left eye. There were $3+$ diffuse vitreous cells bilaterally and small patches of yellowish subretinal pigment epithelial infiltrates bilaterally. A vitreous biopsy was performed on the left eye and was negative. At the time of the vitreous biopsy, $1 \mathrm{mg}$ of rituximab was injected into the left eye. The biopsy was acellular and, therefore, negative. One week later, $1 \mathrm{mg}$ of intravitreal rituximab was given in the right eye. The patient was followed up closely and he underwent systemic treatment with high-dose methotrexate. On last examination, approximately 3 months after his intravitreal injections of rituximab, the patient's visual acuity with correction was $20 / 20$ and 20/400 in the right and left eye, respectively. There was only trace to $1+$ vitreous cell bilaterally on examination. There was no clinical evidence of toxicity.

\section{Case 3}

A 66-year-old man presented with blurred vision to the Ophthalmology Department. He had a known history of diffuse large B-cell lymphoma 3 years ago that had been treated with radiation and $\mathrm{CHOP}$ chemotherapy. About 1 year after the treatment, the patient had a seizure and was found to have CNS involvement with diffuse large B-cell lymphoma. The patient was treated with whole-brain radiation.

On examination, the patient's visual acuity with correction was 20/200 on the right eye and 20/200 with pinhole to $20 / 60$ on the left eye. There were $3+$ diffuse, large vitreous cells in the right eye with multifocal, elevated subretinal infiltrates temporal to the macula. The left eye had no vitreous cell or choroidal lesions. The patient's right eye underwent pars plana vitrectomy, vitreous biopsy, subretinal biopsy of the subretinal pigment epithelial, and endolaser. The patient was also given $1 \mathrm{mg} / 0.1 \mathrm{ml}$ of rituximab intravitreally to the right eye at the time of surgery. The subretinal biopsy and vitreous biopsy showed large B-cell lymphoma. The patient was started on high-dose methotrexate for treatment of primary CNS lymphoma. On last examination, 2 months after surgery, the patient's visual acuity with correction was 20/100 with pinhole to $20 / 30$ on the right eye and 20/40 pinhole to $20 / 30$ on the left eye. There were no vitreous cells on examination nor were there any signs of clinical toxicity of the intravitreal rituximab.

\section{Discussion}

A recently published study showed that the half-life of $1 \mathrm{mg}$ of rituximab injected into the vitreous cavity of rabbits was 4.7 days. ${ }^{4}$ The levels remained above $10 \mathrm{ng} / \mathrm{ml}$ for 72 days. The authors also demonstrated no ocular toxicity in four treated rabbits. They recommended more data to confirm their findings. We also found no histologic evidence of toxicity of $1 \mathrm{mg}$ of rituximab in the rabbits we studied. These two separate studies now give more evidence that one injection of rituximab does not cause toxicity in rabbit eyes. In addition, we found no toxicity in five eyes of three patients injected with $1 \mathrm{mg}$ of rituximab. Other humanized antibodies including bevacizumab and ranibizumab have also shown no significant ocular toxicity in doses that have been locally effective. A limitation to this study is that toxicity was determined only by light microscopy, and ERGs and electron microscopy were not performed in the animals (or humans) that have undergone injections. Further studies and follow-up are indicated.

The vitrectomy performed in two of the five eyes could have decreased the half-life of rituximab. Alternatively, it could also increase the exposure of the macula to rituximab, similar to the findings of intraocular gentamicin, which was found to have the same, if not greater, toxicity in vitrectomized eyes compared with nonvitrectomized eyes; but thus far, no evidence of toxicity has been noted. ${ }^{5,6}$ Rituximab has also been used 
intrathecally with apparently low intracerebral neurotoxicity. ${ }^{2}$ A recent study confirms the ability of rituximab to penetrate all layers of the rabbit retina. ${ }^{7}$

In summary, our data support the finding by Kim et $a l^{4}$ regarding the lack of toxicity of intravitreal rituximab in animal eyes and presents the initial findings of lack of toxicity of intravitreal rituximab in human eyes. Since these patients all underwent either additional ocular treatment (ie intraocular methotrexate or surgery) or additional systemic treatment of their lymphoma, we cannot make conclusions about the clinical effectiveness of intraocular rituximab. Further studies of intraocular rituximab are necessary to confirm the lack of toxicity at this dose and whether it is effective local therapy in eyes with intraocular lymphoma.

\section{Acknowledgements}

This study was supported in part by an unrestricted grant from Research to Prevent Blindness Inc., NY. This paper was presented as a talk at the American Uveitis Society Annual Meeting, Fort Lauderdale, Florida, April 29, 2006.

Conflict of interest: None of the authors have any proprietary interest in any aspect of this report.

\section{References}

1 Pels H, Schulz H, Schlegel U, Engert A. Treatment of CNS lymphoma with the anti-CD20 antibody rituximab: experience with two cases and review of the literature. Onkologie 2003; 26(4): 351-354.

2 Enting RH, Demopoulos A, DeAngelis LM, Abrey LE. Salvage therapy for primary CNS lymphoma with a combination of rituximab and temozolomide. Neurology 2004; 63(5): 901-903.

3 Fishburne BC, Wilson DJ, Rosenbaum JT, Neuwelt EA. Intravitreal methotrexate as an adjunctive treatment of intraocular lymphoma. Arch Ophthalmol 1997; 115(9): 1152-1156.

4 Kim H, Csaky KG, Chan CC, Bungay PM, Lutz RJ, Dedrick RL et al. The pharmacokinetics of rituximab following an intravitreal injection. Exp Eye Res 2006; 82: 760-766.

5 Rosenbaum JD, Krumholz DM, Metz DM. Gentamicin retinal toxicity after cataract surgery in an eye that underwent vitrectomy. Ophthalmic Surg Lasers 1997; 28(3): 236-238.

6 Talamo JH, D’Amico DJ, Hanninen LA, Kenyon KR, Shanks ET. The influence of aphakia and vitrectomy on experimental retinal toxicity of aminoglycoside antibiotics. Am J Ophthalmol 1985; 100(6): 840-847.

7 Pulido JS, Shukla D, Bakri SJ et al. Rituximab penetrates full thickness retina in contrast to tissue plasminogen activator. Retina (In press). 